$$
\text { "tmcs-vajda" — 2012/12/3 - 11:56 — page } 389 \text { — \#1 }
$$

\title{
Learning and teaching combinatorics with Sage
}

\author{
ISTVÁN VAJDA
}

Abstract. Learning Mathematics is not an easy task, since this subject works with especially abstract concepts and sophisticated deductions. Many students lose their interest in the subject due to lack of success. Computer algebra systems (CAS) provide new ways of learning and teaching Mathematics. Numerous teachers use them to demonstrate concepts, deductions and algorithms and to make learning process more interesting especially in higher education. It is an even more efficient way to improve the learning process, if students can use the system themselves, which helps them to practice the curriculum.

Sage is a free, open-source math software system that supports research and teaching algebra, analysis, geometry, number theory, cryptography, numerical computation, and related areas. I have been using it for several years to aid the instruction of Discrete Mathematics at Óbuda University. In this article I show some examples how representations provided by this system can help in teaching combinatorics.

Key words and phrases: computer algebra systems in learning mathematics.

ZDM Subject Classification: U70, K20.

\section{Introduction}

Learning mathematics is not an easy task for most of the students, since this subject works with especially abstract concepts and sophisticated deduction. According to Skemp the particular problem (but also the power) of mathematics lies in its great abstractness and generality, which are achieved by particularly intelligent individuals of successive generations each of whom has been abstracting from, or generalizing, concepts of earlier generations [9].

Copyright (c) 2012 by University of Debrecen 


$$
\text { "tmcs-vajda" — 2012/12/3 - 11:56 — page } 390 \text { — \#2 }
$$

Some experts of mathematics education distinguish five strands of proficiency, which students have to develop during their learning process [3]:

- conceptual understanding - comprehension of mathematical concepts, operations, and relations,

- procedural fluency - skill in carrying out procedures flexibly, accurately, efficiently, and appropriately,

- strategic competence - ability to formulate, represent, and solve mathematical problems,

- adaptive reasoning - capacity for logical thought, reflection, explanation, and justification,

- productive disposition - habitual inclination to see mathematics as sensible, useful, and worthwhile, coupled with a belief in diligence and one's own efficacy.

While many students in higher education lack a notable part of the above skills, computer algebra systems (CAS) brought new opportunities into math instruction, altering learning and teaching methods. Though there is a risk that using CAS can generate the solutions of the exercises without students' understanding of the related concepts and algorithms [8], properly used they can facilitate some of the above skills as the conceptual understanding and productive disposition of students while altering the idea of others as procedural fluency [4].

The following features often considered as the most important advantages of using CAS in math education:

- It is a tool that enables us to present more representations of the examined objects.

- Graphical components can visualize complicated concepts.

- The long and tedious numeric calculation can be processed by the computer, so students can concentrate on the concepts and associations.

- It allows us to present more realistic examples.

Authors usually show several examples how to use the graphical components since they are very helpful in some topics of mathematics like calculus or graph theory [1] [4]. In this article I render the role of representations provided by CAS in teaching combinatorics. Examples presented in sections 3-4 were tested in my computer aided experimental courses at Óbuda University. 


$$
\text { "tmcs-vajda" — 2012/12/3 - 11:56 — page } 391 \text { — \#3 }
$$

\section{About the experimental courses}

Most of the articles discuss how to use CAS in teaching calculus or elementary algebra i.e. [6][2][7], while other topics are yet to explore in this regard. Discrete Mathematics is one of them, since it consists of many different topics that require dissimilar teaching methods and often cannot be illustrated visually. Since other researchers suggest that using CAS improves the achievement of students' skills and understanding in other topics, I offered courses of the subject in computer laboratory since 2009 September at Óbuda University. This experiment affects two study groups per semester. The aim of the course is to develop students' problem solving skills and mathematical understanding, furthermore provide a useful tool to help their studies.

Though Sage is one of the most recent computer-algebra system it is widely used in teaching mathematics [10]. It includes many other systems, covers many topics of mathematics and is developing rapidly. Since it is a free and open source software product students can use it not only in classes but to prepare their homework too, which is an important advantage over other popular computer algebra systems. Since attendees of my courses are computer science and engineering students, they have usually no difficulties with the usage of the system.

Most of the time students work independently in classes but it is often necessary to review some concepts of mathematics and appropriate functions of the system at the beginning. Naturally they can ask questions if some difficulties would arise or allowed to use the help system and their notes. Their homework consists of six problems similar to the ones solved in class. They have to present their solutions in a week and their accomplishments affect their marks at the end of the semester. However the most important parts of the assessment of students' work are the two tests they have to write on the computer.

\section{Representations of permutations}

Permutations are one of the combinatorial concepts that can be represented diversely. Students usually get to know the static concept of permutation at secondary school:

Definition 1. Permutation is an arrangement of a set of objects into a particular order. 
This interpretation harmonizes with the following representation of the Sage program: (Figure 1) However the solution of several math problems requires another definition of permutations.

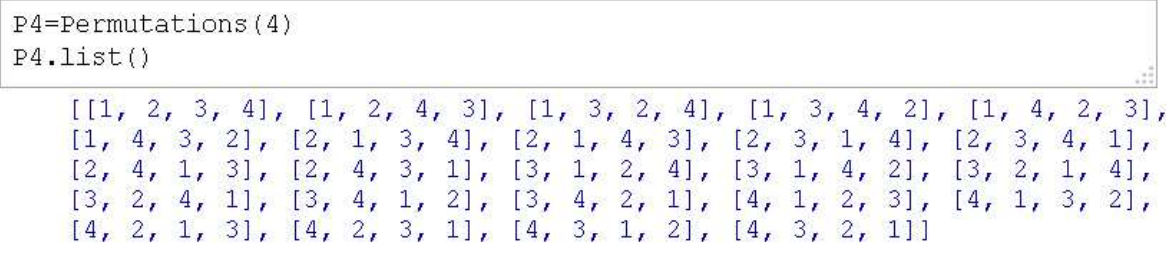

Figure 1. List of permutations

EXAMPLE 1. In how many different ways can five letters be mixed up so that not any of the letters gets to the appropriate recipient?

In order to solve Example 1 [11], students have to understand the concept of fixed points, which needs the precognition of the Definition 2.

Definition 2. Permutation of a finite set $S$ is a bijection from $S$ to itself.

Since this definition is more abstract than Definition 1, students have more difficulties to understand it. In order to help the learning process, we have to show suitable representations of permutations, some of which we can generate via Sage. You can see an example in Figure 2, which displays the connection between the two meanings of permutation.

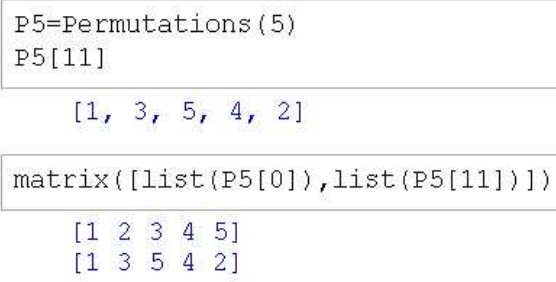

Figure 2. Matrix of a permutation

Figure 3 represents the same, but it emphasizes better that permutations are in fact functions. 


$$
\text { "tmcs-vajda" — 2012/12/3 - 11:56 — page } 393 \text { — \#5 }
$$



Figure 3. Permutation as a function

Definition 3. Let $f: S \rightarrow S$ be a permutation of a set $S$. An element $s \in S$ is a fixed point of $f$ if $f(s)=s$. That is, the fixed points of a permutation are the points not moved by the permutation.

Definition 4. The $f: S \rightarrow S$ permutation is fixed point free, if $\forall x \in$ $S: f(x) \neq x$.

Thus we can rephrase Example 1 using Definitions 3-4:

Example 1* How many $S \rightarrow S$ fixed point free permutations exist, if $|S|=5$ ?

The usual solution applies the inclusion-exclusion principle [11]. Let $N$ denote the number of permutations of five elements and $N_{\alpha_{1} \ldots \alpha_{r}}$ the number of permutations in which $\alpha_{1}, \ldots, \alpha_{r}$ fixed points. (They can have other fixed points too.) The number of fixed point free permutations can be calculated as follows:

$$
\begin{aligned}
& N^{(0)}=N-N_{1}-N_{2}-\ldots-N_{5}+N_{12}+\ldots+N_{45}- \\
& -N_{123}-\ldots-N_{345}+N_{1234}+\ldots+N_{2345}-N_{12345}= \\
& \quad=5 !-\left(\begin{array}{l}
5 \\
1
\end{array}\right) 4 !+\left(\begin{array}{l}
5 \\
2
\end{array}\right) 3 !-\left(\begin{array}{l}
5 \\
3
\end{array}\right) 2 !+\left(\begin{array}{l}
5 \\
4
\end{array}\right) 1 !-\left(\begin{array}{l}
5 \\
5
\end{array}\right) 0 !=44
\end{aligned}
$$

This solution is usually hard to understand for the students, because of the usage of so many subsets of permutations. It becomes easier if they study these subsets that can be generated by Sage. In Figure 4 we can see all of those permutations of $\{1,2,3,4,5\}$ in which 2 and 4 are fixed points, but four of them have others too. Apparently $N_{24}=6$. 


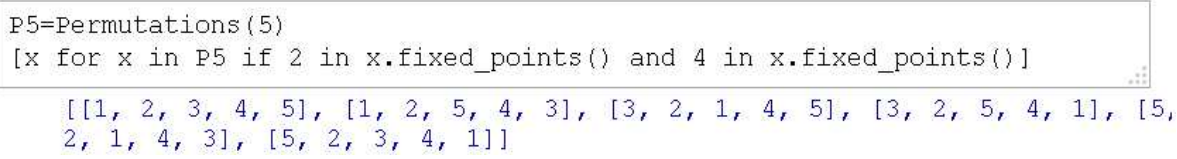

Figure 4. Special permutations

Another solution arises from the cycle notation of permutations, which expresses the permutation as a product of disjoint cycles. For example $[3,1,4,2,5]$ consists of two cycles $(1,3,4,2)$ and (5), which means that $1 \mapsto 3,3 \mapsto 4,4 \mapsto 2$, $2 \mapsto 1$ and $5 \mapsto 5$. (Figure 5) Fixed points compose cycles with one element, so the solution of Example 1 is the number of permutations without a cycle of one element. So the adequate permutations consist of one cycle of five elements, or two cycles with two and three elements respectively. So the number of the fixed point free permutations is:

$$
4 !+\left(\begin{array}{l}
5 \\
3
\end{array}\right) \cdot 2=44
$$

Permutation $([3,1,4,2,5])$.to_cycles()

$[(1,3,4,2),(5)$,

Figure 5. Cycle notation

The solution of Example 1 can be easily produced by Sage since the derangements() function generates the fixed point free permutations of a set, while the number_of_derangements() function returns the number of them.

EXAMPle 2. Let $S$ be given a set of 10 elements. Determine the number of those permutations of $S$ which have exactly 4 fixed points!

Solution: There are $\left(\begin{array}{c}10 \\ 4\end{array}\right)$ ways to choose the four fixed points. Among the other six elements there cannot be further fixed points. Six elements have 265 fixed point free permutations, so the number of the permutations in question is $\left(\begin{array}{c}10 \\ 4\end{array}\right) \cdot 265=55650$. (Figure 6)

While students are usually familiar with Pascal's triangle and the $\left(\begin{array}{l}n \\ k\end{array}\right)=$ $\frac{n !}{k !(n-k) !}$ equality, so they can calculate the binomial coefficients, it would be difficult to produce the number of derangements for them without a sufficient computer algebra system. Some of them even prefer the logically simpler way 


$$
\text { "tmcs-vajda" — 2012/12/3 - 11:56 — page } 395 \text { — \#7 }
$$

number_of_derangements $([1,2,3,4,5,6])$

265

Figure 6. Number of derangements

of counting the appropriate permutations. (Figure 7) Either way the computer enables us to solve math problems that could not be solved conventionally.

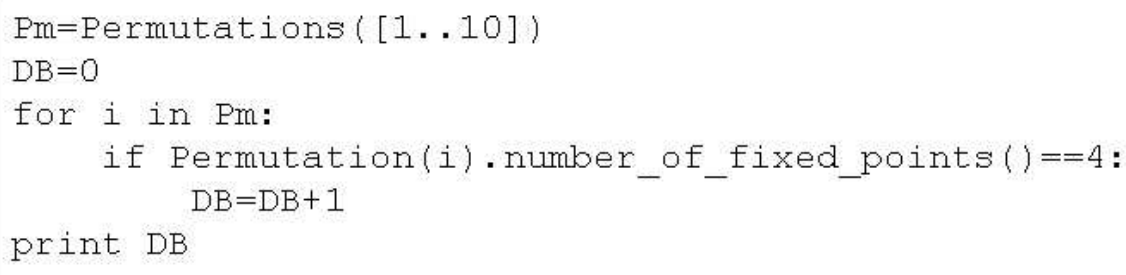

Figure 7. Counting of 4-fixed-points-permutations

\section{Arrangements of Multisets}

Example 3. Peter wants to send postcards to 10 friends. In the shop there are only 3 kinds of postcards, 4, 5 and 6 from each respectively. In how many ways can he send the postcards, if he wants to send one card to each friend?

This example is similar to others that can be found in several books [11] [5], but usually we do not know how many postcards are available, only that there is a large number of each kind. If that would be the case, we had three ways to choose a postcard for each person, so the solution would be $3^{10}=59049$. Contrarily Example 3 cannot be solved in some familiar way due to the included conditions, however we can easily generate the answer via Sage. (Figure 8)

Though Example 3 is not suitable to improve the problem solving skill of students in a conventional way, since the Sage system makes the hard work, it can improve the learning process of students nonetheless. Firstly such problems provide an appropriate way to introduce new concepts, in this case the concept of multiset. Secondly if students try to find a solution without the computer, they 
Figure 8. Number of arrangements

can achieve a deeper understanding of combinatorial calculations, going over why they do not work in this example.

\section{Students' achievement and opinion}

Students of Discrete Mathematics courses at Óbuda University have to undergo two tests per semester, participants of the experimental groups work on computer while the others sit the conventional written examination. Usually there are only one or two questions related to combinatorics in a semester because of the several topics to check. The students' achievements of the last three years are summarised in Table 1.

Table 1. Students' accomplishment in combinatorics questions

\begin{tabular}{lcc}
\hline & Experimental groups & Control groups \\
\hline Number of questions & 8 & 6 \\
Number of students & 41 & 52 \\
Number of solutions & 294 & 269 \\
Students' achievement (\%) & 62 & 34 \\
\hline
\end{tabular}

To learn the students' opinion about the experiment, I asked them to fill a questionnaire at the end of the course. Most of them considered the course successful, only 3 students of the 92 preferred the conventional way of teaching. About half of them think that combinatorics is one of the most difficult topics of Discrete Mathematics despite of the relatively good result they achieved. 


$$
\text { "tmcs-vajda" — 2012/12/3 — 11:56 — page } 397 \text { — \#9 }
$$

\section{Conclusion}

Discrete Mathematics has many topics, which require different thinking and teaching methods. The Sage system has many facilities which can help to understand several concepts and methods of the subject, but not all of them. Graphical components can visualize graphs and related concepts as posets or groups, but its function is not so overall, than in teaching calculus.

However Sage can represent concepts in many other ways as the above examples demonstrate at the topic of combinatorics, which helps to understand them more thoroughly. Another advantage of the system is, that it can manage calculations, which would be too complicated and tedious otherwise, expanding the range of solvable problems. This often allows us to introduce new concepts in a serried way and enables us to work with more realistic examples.

The development of the aforementioned five strands of proficiency can be aided more efficiently by reasoned usage of CAS. Students' grades imply a deeper understanding and strategic competence, though the meaning of the latter is slightly modified. Procedural fluency changes even more.

The bigger alteration is the classwork of students, which improved dramatically in the computer laboratory. While they require the help of the teacher yet, their work is more self-supporting getting more information from the computer and each other. The betterment of their productive disposition is the most noticeable change induced by the usage of the system.

\section{References}

[1] M. Aktümen and T. Kabaca, Constructing the disk method formula for the volume obtained by revolving a curve around an axis with the help of CAS, Teaching Mathematics and Computer Science 5, no. 2 (2007), 363-376.

[2] J. Bradley, M. Kemp and B. Kissane, Understanding what you are doing: A new angle on CAS, in: Communications of Remarkable Delta'03 Fourth Southern Hemisphere Symposium on Undergraduate Mathematics and Statistics Teaching and Learning, 2003, 50-64.

[3] J. Kilpatrick, J. Swafford, and B. Findell, editors, Adding it up: Helping children learn mathematics, National Academy Press, 2001.

[4] Z. Kovács, Blind versus wise use of CAS, Teaching Mathematics and Computer Science 5, no. 2 (2007), 407-417.

[5] L. Lovász, J. Pelikán and K. Vesztergombi, Discrete Mathematics, Springer, 2003. 


$$
\text { "tmcs-vajda" — 2012/12/3 - 11:56 - page } 398 \text { - \#10 }
$$

[6] K. A. Nabb, CAS as a restructing tool in mathematics education, in: Proceedings of the $22^{\text {nd }}$ International Conference on Technology in Collegiate Mathematics, 2010.

[7] R. Pierce and K. Stacey, Observations on students' responses to learning in a CAS environment, Mathematics Education Research Journal 13, no. 1 (2001), 28-46.

[8] H. Povey and M. Ransom, Some undergraduate students' perceptions of using technology for mathematics: Tales of resistance, International Journal of Computers for Mathematical Learning 5, no. 1 (2000), 47-63.

[9] R. Skemp, The Psychology of Learning Mathematics, Lawrence Erlbaum Associates, 1987.

[10] Teaching with sage, http://wiki.sagemath.org/Teaching_with_Sage.

[11] N. J. Vilenkin, Kombinatorika, Műszaki könyvkiadó, Budapest, 1971.

ISTVÁN VAJDA

ÓBUDA UNIVERSITY

HUNGARY

E-mail: vajda.istvan@nik.uni-obuda.hu

(Received February, 2012) 